\title{
The Italian Public Administration Electronic Market: Scenario, Operation, Trends
}

\author{
Danilo Broggi ${ }^{1}$ \\ ${ }^{1}$ Consip - Chief Executive Officer, Italy, danilo.broggi@tesoro.it
}

\begin{abstract}
A critical element that has been generally recognized is that the Italian Public Administration provides services and operates emphasizing the formal abidance to administrative regulations and procedures, having in part lost sight of its institutional goal of providing quality and timely services to citizens and -so crucial in our digital age- the goal of "system creation". The opportunity that Italy is given through eGovernment lies in fact in the possibility of promoting a change management of public services towards a culture aimed at the satisfaction of the final user. From this point of view, eProcurement can become the "Trojan Horse" (or the killer application) capable of promoting change within Public Administration. This is true, first of all, because it synthesizes all the critical aspects of typical change management processes: Structure reorganization, integration and interoperability of functions/service, process re-engineering, professional training and growth of human resources, legislative revision, technology introduction, the monitoring of public expenditure and its monitoring (while maintaining the decentralization of expenditure decisions), as well as quality control. Secondly, because it is an issue that covers horizontally all Public Administrations, whose boundaries and procedures are well known and whose achievable results are therefore highly visible and economically valuable. Thirdly, the contracting sector appears more conducive than others to the promotion of this change process because it has the necessary critical mass in terms of expenditure volume to support innovation. Public contracts account for more than $16 \%$ of GNP. Lastly, through a growth in the efficiency of public contracts it is possible to achieve results that are substantial and permanent in the areas of process savings, good and services cost savings and of positive externalities in terms of tool innovation that will promote market innovation
\end{abstract}

Keywords: eGovernment, eProcurement, Public Administration Electronic Market (MEPA) 


\section{Defining eProcurement}

Considering the entire supply value chain, eProcurement tools, those employed in electronic search-selection-purchase, may be implemented from the requirement manifestation phase to the invoicing and payment management phase. More specifically, if one considers the electronic tools employed by public authorities to select their contractors, one may employ the term ePublicProcurement.

Public eProcurement may be defined as the set of technologies, procedures, operations and organizational modalities that allow for the on-line selection and provisioning of goods and services through the opportunities offered by the development of the Internet and electronic commerce. Public eProcurement is obviously influenced by the different structure that characterizes the contract categories related to the specific role played by Public Administration within the legislative system as a whole.

Consequently, the areas of intervention where the introduction of eProcurement within a public entity may have a significant impact are essentially four: Organization, processes, technology and public expenditure.

At the international organization level, the past ten years have witnessed the emergence of a strong interest in themes related to eGovernment and electronic purchasing tools. More specifically, at the EU level there is a strong interest in eProcurement and there have been at least three relevant initiatives on the part of the Directorates General of the European Commission (Internal Market, Information Society and media IDABC, Enterprise and Industry European eBusiness Support Network).

Regarding the Italian scenario, the electronic purchasing tools that are most common in both the Central Public Administration (all managed exclusively by Consip) and at the regional and local levels (managed by ad hoc purchasing agencies or by administrative departments), essentially deal with the search, comparison and supplier and product selection phases. We are talking about electronic shops, electronic tenders and electronic marketplaces.

Compared to electronic tenders and auctions (on-line contractor selection procedures that allow for different sorts of tenders, among them the sealed envelope with the most economically convenient offer), and electronic shops (eShops - "static" eProcurement tools that publish on-line pre-negotiated catalogues where one may shop selecting quantities, with only a limited offer of different options), eMarketplaces are undoubtedly eProcurement's most innovative, flexible and complete tool.

Apart from these tools, the Directive n. 2004/18 and the Legislative Decree n. 163/06 have introduced in Italy the Dynamic Purchasing System, a tool which has yet to be employed that may be described as a totally electronic acquisition process for normal everyday purchases (goods/services with characteristics that are generally available in the market). Always accessible by suppliers, but limited in time (maximum four years), with indicative offers (through on-line catalogues) 
that may be dynamically improved. This tool is also employable in purchases that are above the community threshold.

In general terms, it is clear that the benefits of the use of eProcurement are more interesting if it is backed by is a strategic approach, in which the commitment and the goals are well defined, where there has been an investment in training and change management of the purchases function and where there has been adequate communication with suppliers.

Regarding specifically transparency, direct empirical experience shows that the use of electronic purchasing procedures leads to a growth trend due to various elements. Briefly, these are: 1) A general and uniform access to information without discriminations. 2) The standardization of procedures. 3) The tracking and monitoring of all contacts, interactions and document exchanges between parties.

4) The minimizing of discretional action in the evaluation phases .

As far as public authorities are concerned, the advantages of eProcurement are directly related to the on-line tools themselves, as well as to the operational framework of eProcurement in the wider sense of the term.

Among the other advantages, which must in any case be interpreted in a systematic approach, one may mention the acceleration of procedures, the reduction of the time spent in purchasing processes, the reduction of the cost of managing tenders, the simplification of processes, the direct and constant monitoring of public expenditure, the professional growth of the personnel in charge of the processes, the increase in the potential pool of suppliers, the savings in prices, the de-materialization of documentation and the possibility to implement digital document management projects.

Both from a first-hand field experience and from a sample-based analysis, what emerges is a number of critical factors that represent real obstacles to the adoption of eProcurement on the part of Italy's PA, as well as a number of false truths.

Among the critical factors, it is important to underline the fact that, apart from the technological obstacles, there are also the typical problems related to an incomplete and ineffective change management program within the Public Administration. More specifically:

- The absence of a clear strategic vision for public eProcurement that is clearly and effectively communicated, that does not raise concerns over long-term strategies and that does not encourage the creation of legislative hiccups that lead to confusion both in the area of demand and in the area of supply.

- The absence of implementing regulations.

- The absence of both an adequate level of training and of a recognition of new professional qualifications among civil servants (e.g., the eBuyer).

- The absence of a monitoring and evaluation activity, as well as of a rewarding system for the Public Administrations and managers that make use of these tools.

- The existence of the digital divide non only as a concept, but also as a real technological barrier (not all Public Administrations have even basic electronic mail and very few use digital signatures). 
- The fact that eProcurement platforms sometimes are not stable and often are not all that simple and clear-cut to use.

- The fact that the supply side is hesitant to join in, especially at the local level, fearing a greater competition, if it does not understand that eProcurement is a mandatory path to take in the long run in order to sell one's products to the PA.

Remaining within the organizational and technological framework, it must be recognized that the adoption of eProcurement platforms, especially if they are not open source, is costly and what is even more so is the management of the backoffice (databases and on-line supplier catalogues). The solutions offered by the public central purchasing bodies overcome this problem through their free delivery of solutions to all Public Administrations. It would obviously be helpful if it were possible to share organizational and technological standards in order to promote the interoperability of data and to avoid creating digital barriers in the local markets.

\section{The Public Administration Electronic Market (MEPA)}

The Public Administration Electronic Market (MEPA) is a public eProcurement tool managed by Consip on behalf of the Ministry of the Economy and Finance. It is a dynamic tool (in that it allows suppliers to register, post and change their wares, services and prices at any moment in time) in which products and services are presented in structured catalogues and described according to standard formats. The purchasers are registered Authorities that, after a search and comparison of the products being offered, may implement purchases directly through orders from the catalogues, or by requesting preliminary offers. An identical product/service may be sold by various suppliers at different conditions (as in the real market) and there is no pre-negotiation on the part of Consip.

The MEPA is part of the wider Program for the Rationalization of Public Expenditure that was launched in 2000 by the Ministry of the Economy and Finance in the wake of the introduction of a new model for the optimization of public provisioning in Italy.

As mentioned, the MEPA is an electronic market where the Ordering Points (OPs) represented by the individuals that have purchasing authority within their respective Public Administrations may search for, compare between and purchase the goods and services offered by the supplier enterprises that are authorized to display their catalogues on the system, within the constraints set in specific tenders published by Consip for the different product categories. It is therefore a market that is:

- Selective, in that its access and use is reserved to users that have passed a qualification process that is based on the verification of the possession of specific requirements. 
- Specialized, in that it is aimed at meeting the procedural and administrative requirements that are specific to the provisioning function of Public Administrations and of the companies that interact with them as suppliers (document characteristics, filing procedures, use of digital signatures, etc...).

- Based on a catalogue of authorized products, in that all the commercial transactions that take place in the market deal with goods/services offered by suppliers in catalogue form and displayed on the system following an authorization process that is managed by Consip.

- Usable only for purchases of quantities of goods and services that are valued below the community threshold.

Suppliers may decide where to sell, starting either from a local dimension (their Province), or aiming for a regional range of action, to the option of offering their products/services in the whole of Italy. In this aspect, the system is flexible in that it allows for choices among different operational dimensions (that can be dynamically modified) and between different user characteristics (Public Administrations and Suppliers), that may generate personalized "geographical visits" (ranging from the individual Province to the whole national territory) on the basis of their requirements and capabilities.

Even though it is a highly innovative tool, the MEPA does not change the legislative and commercial regulations that characterize the purchasing processes of the Public Administration, specifically safeguarding the importance of purchaser-supplier relations, especially where the "localization" of the service is a significant component.

The authorization of suppliers aiming to join the MEPA takes place through specific authorization Tenders that are published for all the product and service categories that are sold on the Electronic Market.

The authorization procedure deals both with the suppliers and the products and services that they would like to offer to Public Administrations. In order to offer Public Administrations a qualified list of suppliers and wares, enterprises must have the minimal requirements necessary to prove that they have the professional, economic and financial capacity that is required in the authorization tender and may offer in the system only the goods/services that meet the requirements that are set in the relevant Technical Specifications. The permanence of a supplier in the MEPA guarantees Public Administrations that its statements have been verified and approved by an ad hoc Technical Committee every six months. Apart from a products and services search engine and an electronic filing system, the Electronic Market offers registered authorities the possibility to process contracts through two different purchasing procedures:

- Direct Order: The Administration may purchase goods and services directly through the catalogues of the suppliers that are registered in the Electronic Market. The publication of a catalogue on the part of the supplier amounts to a public offer reserved to the Administrations that are registered in the Electronic Market. 
- Offer Request: This allows the Administration to request from registered suppliers various and diverse offers related to the products and services registered in the Electronic Market, thus allowing them to meet more specific requirements. In simpler terms, this procedure may be used to request more than one preliminary offer from various suppliers on the same product/service, thus putting them in competition and trying to obtain conditions that are more favourable than those offered in the catalogue.

The advantages brought by the use of the MEPA, apart from the general benefits of eProcurement, may include not only cost and time savings in the provisioning cycle, but also changes that the use of the tool might bring within an organization. As far as the price savings obtained by the Public Administrations are concerned, MEPA's characteristics make for a very complex evaluation.

The heterogeneous and diversified nature of the products and services offered on the MEPA and the different selection modalities that are available (through direct catalogue order, or through offer requests) make it very difficult to identify a general compared analysis model between the purchasing prices obtained by the electronic tool and those obtained by Public Administrations with other methods. Nonetheless, a number of perception polls (seminars, conferences, interviews, etc...) of many Public Administrations that have so far used the MEPA tend to confirm the existence of an economic advantage in terms of price reduction, related especially to a more open market and, consequentially, to greater competition and transparency. As has emerged in analyses made on private business to business eMarketplaces, the decision to introduce these tools is aimed ad producing process savings, even though the average cost savings on the prices of goods/services is estimated to be around 8 per cent.

The full deployment of the benefits that have been mentioned above requires a medium to long-period timeframe, both because the results and the benefits of the use of the Electronic Market are often not immediately felt by the users, but rather manifest themselves in the long run as the user becomes more proficient with the tool, and because there are still some high hurdles to overcome in terms of culture, technology, legislation and administrative structure before one can achieve a complete dissemination of the tool.

It is important to underline the fact that the Public Administration Electronic Market -MEPA- provides a stimulus towards innovation not only for Public Administrations, but also for enterprises, especially the small and medium-sized companies. In these years of dissemination of the MEPA, it has become clear how the use of the tool has brought about benefits in terms of transparency, change management and process savings not only for Public Administrations, but also for the enterprises participating in the program.

Undoubtedly, the participation in the MEPA amounts to the activation of a new channel of commercial relations with the Public Administration -at a limited cost, given that participation in the Electronic Market is free of charge- as well as the extension of the potential market in the context of Public Administrations, both at a national and at a local level. For many companies, the tool guarantees a nation- 
wide visibility of their offer as well as a greater penetration in their local territory, without requiring further commercial investments.

For the companies that operate locally, the MEPA provides a greater competitiveness related to the localization and/or specialization of their offer that allows to highlight the added value and services provided by local small and medium-sized enterprises. Furthermore, the presence of the MEPA provides a better knowledge of the offer available within the product category of interest and an optimization of one's positioning with regards to the competition.

One must also not forget that the pre-set rules and procedures that are shared by all actors involved in the Electronic Market amount to a guarantee of greater transparency in tender procedures. Through the workings of the market, the presence of suppliers in the MEPA has the collateral effect of generating a spontaneous self-regulation of the system of supply in which "all see and compare themselves to all", making for a greater fairness in competition through transparent procedures.

A further benefit for companies is represented by the possibility of a continuous updating of their offer due to the dynamic nature of the catalogues. This allows suppliers to implement specific commercial strategies that may be aimed at reducing overstocking or at saturating productive capacity. Compared to more traditional supply modalities, the dynamism of catalogues allows to constantly update the supply and to offer in real time promotions in terms of both prices and new product lines. This opportunity is especially taken advantage of in markets that are characterized by products with a high rate of obsolescence.

Another benefit of the use of this technology is given by the lower sales costs brought about by the reduction of the cost of intermediation and of managing the entire sales process. Many companies have made accurate analyses of the costs and savings obtained through their participation in the sales procedures processed through the MEPA.

A last point that should be made is that which relates to the sharing of documentation and know-how. The "packaging" of commercial, administrative and category-related competences provided by the MEPA through its on-line catalogues and the documentation on tenders and technical specification models represents one of principal benefits introduced by the MEPA, both for enterprises and Public Administrations. The documentation's characteristics of completeness and clarity support suppliers in the definition of their offer and makes programming and reporting on the service easier, strongly impacting on the quality of the service that is offered. Furthermore, the possibility given to Public Administrations to use technical specifications and supply conditions that may be personalized according to specific requirements focalizes a greater attention on higher supply standards and a greater guarantee of the quality of supply.

That said, companies, especially the smaller ones, have to overcome some "difficulties", ranging from technical hurdles, to cultural obstacles to organizational ones. 
From the point of view of the product category, meaning the nature of the products and services that are negotiable on the system, the MEPA is characterized as a general spending tool.

The selection of the product categories on offer is aimed at meeting the most common goods and services purchasing requirements of Public Administrations and offers a balance between range (the variety of goods/services categories) and depth (the assortment of goods/services in the same category) of choice.

The main characteristics that make a product category appropriate for its activation on the MEPA are a medium-high comprehensive frequency of purchase, a low complexity of its specifications, a "strategic" relevance of the demand/supply, its complementarity with other public administration purchasing tools (e.g,, the framework contracts), a prevalence of orders below the community threshold and a wide-ranging supply in terms of numbers of suppliers.

The growth and consolidation of an electronic market requires the so-called "crossed-network effect", meaning the creation of a population level of both the supply and demand sides that is capable of fostering further participation and interest on the part of other companies, and consequently also other Public Administrations.

In these years of dissemination of the tool, it has clearly emerged that the growth in demand is the one dominant factor determining the expansion of the Electronic Market. Companies are in fact very reactive to the perception of the level of dissemination and acceptation of the tool on the part of the Public Administration.

\section{Conclusions}

Both at a European and international level, whether within the wider context of eGovernment programs or the more narrow framework of public expenditure rationalization, eProcurement nowadays has taken on a key role in the efforts aimed at the modernization and increased efficiency of Public Administration, as well as at the monitoring of public accounts. The significance and impact that electronic purchasing tools can have derives not only from the fact that public contracts account for 16 per cent of GNP (EU average), but also from the advantages that they bring to Public Administration change management programs.

The Public Administration Electronic Market (MEPA) is undoubtedly one of the most advanced B2G tools around and is recognized as a best practice at the European level.

As is common with many innovative and relatively new projects, apart from the benefits it provides, the MEPA also has a number of critical elements that slow its dissemination. 
Specifically, these have been highlighted as a) from the demand side, the problem of digital divide (tied to the inadequate spread of the Internet and of the digital signature) and the inertia of users from the point of view or organizational and cultural change management, and b) from the supply side, a reluctance, especially at the local level, to join the game and play by rules that are more competitive, with the risk of losing some traditional "market niches", as well as the aversion to investing in eCommerce without a clear vision of its long-term returns.

Among the advantages provided by the MEPA, apart from those typical of all eProcurement tools (foremost among them a greater transparency), one should highlight process savings in terms of man/hours compared to traditional contracting methods and the possibility of a detailed and constant monitoring of public expenditure (with the added possibility of a compared analysis of expenditure between different Public Administrations).

Among the theoretical issues that the project has evidenced one should not forget the positive reciprocal externalities between demand and supply and the risks of a digital segmentation of markets brought about by a lack of interoperability between different eProcurement systems.

From what has been detailed so far, it is clear that eProcurement tools like the MEPA may act as a catalyst for an organizational and procedural change within the PA that could impact and offer new opportunities on both the public demand and supply sides.

The scenario that has been traced highlights the strategic role that the Public Administration Electronic Market has acquired in the area of below-threshold purchases for the Public Administration at large. This role is further enhanced by the introduction and development of new purchasing tools, such as the Framework Agreements and the Dynamic Purchasing System.

More specifically, the Dynamic Purchasing System may expand the offer of the MEPA in some product categories, allowing the Public Administrations to take advantage of this expansion whenever the adjudication of contracts has to take place according to open procedures (both below and above the community threshold). Furthermore, the Dynamic Purchasing System presents a working model and some critical success factors that are very similar to those that characterize the MEPA, such as the need to guarantee adequate service levels of the purchasing system in organizational and technological terms, the "tertiarity" of its manager with regards to the negotiating activity of operators of the system and the overall efficiency of the new tool in terms of costs and benefits.

The know-how and the experience that have been acquired on the MEPA by all involved actors - Public Administrations, enterprises, industrial associations and Consip- will facilitate the introduction and use of the new tools.

These developments may call for an evolution of Consip's role from that of a contracting body to that of market maker, because -similarly to what happens within the MEPA- Consip would not be playing the role of a "pure" and simple contracting body (as it does in the context of Framework Contracts), but rather, with variations according to the tools being used (MEPA, Framework 
Agreements, Framework Contracts, Dynamic Purchasing Systems), that of manager of a market whose actors operate, from a negotiating point of view, with various degrees of autonomy and thus with variable levels of personalization of provisioning with regards to their needs.

Within this role and on the back of its experience in the planning, management and implementation of new purchasing tools and modalities, the National Purchasing Agency could take on, within the context of the network system to be set up with the regional and local purchasing bodies, a service and "facilitator" role in the promotion of an efficient model in which the purchasing entities and the individual Public Administrations may implement their own electronic purchasing procedures, both above and below the threshold, in a simplified and structured fashion. 